\title{
Finite Sum Representations for Partial Derivatives of Special Functions with Respect to Parameters
}

\author{
By R. G. Buschman
}

\begin{abstract}
The Mellin transformation is used as a method for discovery of cases where the partial derivatives with respect to parameters for certain Whittaker and Bessel functions can be expressed in terms of finite sums involving these functions. These results are easily generalized to the $G$-function, from which, by specialization, formulas involving hypergeometric and other functions can be obtained.
\end{abstract}

1. Introduction. In a recent paper, Laurenzi [4] obtained finite sum expansions for the partial derivative of $W_{\kappa, 1 / 2}(z)$ with respect to $\kappa$ at a positive integral values of $x$ expressed in terms of $W_{l, 1 / 2}(z)$. He also obtained analogs for $M_{x, 1 / 2}(z)$. Similar expressions are known for certain other functions; for example, Abramowitz and Stegun [1] list partial derivatives with respect to order for the Bessel functions $J_{v}, Y_{v}, I_{\nu}$, and $K_{v}$ at nonnegative integral values of $\nu$ and at $\nu= \pm \frac{1}{2}$ and for the Legendre functions $\partial P_{\nu} / \partial \nu$ and $\partial P_{v}^{-1} / \partial \nu$ at $\nu=0$ and $\partial P_{\nu}^{-1} / \partial \nu$ at $\nu=1$. The purpose of our paper is to indicate a method of discovering such formulas and their generalizations by use of the properties of the Mellin transformation along with simple identities involving the $\Gamma$ and $\psi$ functions.

For notation, definitions, and formulas, refer to [1], [2]. The formula for the $\psi$ function, which is of particular use in our manipulations, is

$$
\psi(a+N)-\psi(a)=\sum_{k=1}^{N} \frac{(-1)^{k-1} N !}{k(N-k) !} \frac{\Gamma(a)}{\Gamma(a+k)} .
$$

This comes from formula (30) on p. 19 of [2] where the series terminates if $z=N$, a nonnegative integer. The symbols $j, k, l, m, n, p, q, M, N$ shall represent integers throughout our work.

Our technique of discovery consists of the formal use of the tables of Mellin transforms, manipulations of these transforms with an investigation of those cases where (1) can be used, and the inversion by use of tables. Rigor could then be supplied either by justifying the steps or by induction

Received September 4, 1973.

AMS (MOS) subject classifications (1970). Primary 33A30, 33A35, 33A40, 33A45, 33A50; Secondary 44A15, 44A20.

Key words and phrases. Whittaker functions, Bessel functions, hypergeometric functions, Gegenbauer functions, Legendre functions, $G$-functions, derivative with respect to order, Mellin transformations. 
where recurrence formulas are known, which is the case for many of the special functions of interest. Alternatively, we could work with MellinBarnes integral representations. We illustrate the manipulations for the simple cases and then merely state the generalizations to $G$-functions where they are obtained by directly analogous steps.

2. Partial Derivatives of $W_{\kappa, \mu}(z)$ with Respect to the First Parameter. From the tables of Mellin transforms [3, p. 337, formula (8) ], we have

$$
\mathscr{M}\left\{e^{-x / 2} W_{\kappa, \mu}(x)\right\}=\Gamma\left(\mu+\frac{1}{2}+s\right) \Gamma\left(-\mu+\frac{1}{2}+s\right) / \Gamma(-\kappa+1+s)=g(s),
$$

valid for $\operatorname{Re}(s)>|\operatorname{Re}(\mu)|-\frac{1}{2}$. If we differentiate with respect to the parameter $\kappa$, we obtain

$$
\mathscr{M}\left\{e^{-x / 2}(\partial / \partial \kappa) W_{\kappa, \mu}(x)\right\}=g(s) \psi(-\kappa+1+s) .
$$

Noting also the effect of differentiation with respect to $s$ of a Mellin transform of a function, we have

$$
\begin{aligned}
& \mathscr{M}\left\{e^{-x / 2} W_{x, \mu}(x) \log x\right\} \\
& \quad=g(s)\left(\psi\left(\mu+\frac{1}{2}+s\right)+\psi\left(-\mu+\frac{1}{2}+s\right)-\psi(-\kappa+1+s)\right) .
\end{aligned}
$$

The combination of (3) and (4) gives us

$$
\begin{aligned}
\mathscr{M}\left\{e^{-x / 2}\left((\partial / \partial \kappa) W_{\kappa, \mu}(x)-W_{\kappa, \mu}(x) \log x\right)\right\} & \\
=h(s)=g(s)\left(\psi(-\kappa+1+s)-\psi\left(\mu+\frac{1}{2}+s\right)\right. & \left.+\psi(-\kappa+1+s)-\psi\left(-\mu+\frac{1}{2}+s\right)\right) .
\end{aligned}
$$

If both $\kappa+\mu-\frac{1}{2}$ and $\kappa-\mu-\frac{1}{2}$ are integers, then we can use (1) to express the differences of the $\psi$ functions, which appear in (5), as finite sums. Consequently, we may as well set $\kappa=N / \mathcal{Z}+\frac{1}{2}$ and $\mu=M / 2$ with $M$ and $N$ of the same parity. There are four cases to consider, depending upon the signs of $M+N$ and of $M-N$ (these correspond to the cases of the signs of $c-a-1$ and of $a$ for the hypergeometric function $\Psi(a, c ; x))$.

If $M+N \geqq 0$, the application of (1) allows simplification of the $\Gamma$-function quotient so that, from (2), we have

$$
\begin{aligned}
g(s)(\psi(-N / 2 & \left.\left.+\frac{1}{2}+s\right)-\psi\left(M / 2+\frac{1}{2}+s\right)\right) \\
& =\mathscr{M}\left\{\sum_{k=1}^{N / 2+M / 2} \frac{(-1)^{k}(N / 2+M / 2) !}{k(N / 2+M / 2-k) !} e^{-x / 2} W_{N / 2+1 / 2-k, M / 2}(x)\right\} ;
\end{aligned}
$$

similarly for $N-m \geqq 0$ we have

$$
\begin{aligned}
g(s)(\psi(-N / 2 & \left.\left.+\frac{1}{2}+s\right)-\psi\left(M / 2+\frac{1}{2}+s\right)\right) \\
& =\mathscr{M}\left\{\sum_{k=1}^{N / 2-M / 2} \frac{(-1)^{k}(N / 2-M / 2) !}{k(N / 2-M / 2-k) !} e^{-x / 2} W_{N / 2+1 / 2-k, M / 2}(x)\right\} .
\end{aligned}
$$

Combining (5), (6), and (7), we obtain 


$$
\begin{aligned}
\frac{\partial}{\partial \kappa} W_{\kappa, M / 2}(x) & \left.\right|_{k=N / 2+1 / 2} \\
= & \sum_{k=1}^{N / 2+M / 2} \frac{(-1)^{k}(N / 2+M / 2) !}{k(N / 2+M / 2-k) !} W_{N / 2+1 / 2-k, M / 2}(x) \\
& +\sum_{k=1}^{N / 2-M / 2} \frac{(-1)^{k}(N / 2-M / 2) !}{k(N / 2-M / 2-k) !} W_{N / 2+1 / 2-k, M / 2}(x) \\
& +W_{N / 2+1 / 2, M / 2}(x) \log x
\end{aligned}
$$

for $-N \leqq M \leqq N$ and $M$ and $N$ integers of like parity. Formula (8) reduces to the result of Laurenzi [4, formula (15), p. 131], for $M=1, N=2 n-1$.

On the other hand, if $-(M+N) \geqq 0$, the simplification of (6) does not occur; however, we have

$g(s)\left(\psi\left(-N / 2+\frac{1}{2}+s\right)-\psi\left(M / 2+\frac{1}{2}+s\right)\right)$

$$
\begin{aligned}
= & \sum_{k=1}^{-M / 2-N / 2} \frac{(-1)^{k-1}(-M / 2-N / 2) !}{k(-M / 2-N / 2-k) !} \frac{\Gamma\left(-M / 2+\frac{1}{2}+s\right)}{\Gamma\left(M / 2+\frac{1}{2}+k+s\right)} \frac{\Gamma^{2}\left(M / 2+\frac{1}{2}+s\right)}{\Gamma\left(-N / 2+\frac{1}{2}+s\right)} \\
= & \sum_{k=1}^{-M / 2-N / 2} \frac{(-1)^{k-1}(-M / 2-N / 2) !}{k(-M / 2-N / 2-k) !} \\
& \cdot \mathscr{M}\left\{(-1)^{-M-k} x^{1 / 2-M / 2} D^{-M-k}\left(e^{-x / 2} x^{-1 / 2-k} W_{M / 2+N / 2+1 / 2,0}(x)\right)\right\}
\end{aligned}
$$

where we have used formulas (3) and (9) on p. 307 of [3] combined in the form

$$
\mathscr{M}\left\{-x^{1-\alpha} D\left(x^{\alpha} f(x)\right)\right\}=(s-\alpha) \mathscr{M}\{f(x)\}
$$

to interpret the ratio $\Gamma\left(-M / 2+\frac{1}{2}+s\right) / \Gamma\left(M / 2+\frac{1}{2}+k+s\right)$ and where we have assumed further that $N-M \geqq 0$. Thus, for $M \leqq N \leqq-M$, the first sum of (8) is replaced by

$$
\begin{aligned}
e^{x / 2} \sum_{k=1}^{-M / 2-N / 2} & \frac{(-1)^{-M-1}(-M / 2-N / 2) !}{k(-M / 2-N / 2-k) !} \\
& \cdot x^{1 / 2-M / 2} D^{-M-k}\left(e^{-x / 2} x^{-1 / 2-k} W_{M / 2+N / 2+1 / 2,0}(x)\right) .
\end{aligned}
$$

Further, for $-M \leqq N \leqq M$, the second sum of (8) is analogously replaced by a sum of the form of (11) with $M$ replaced by $-M$. These two forms can be rewritten in several manners by use of the derivative and recurrence formulas on pp. 22,27 , and 28 of [5]; one example of this is to use (2.4.16) to obtain

$$
\begin{aligned}
D^{-M-k}( & \left.x^{-k} e^{-x / 2} x^{-1 / 2} W_{M / 2+N / 2+1 / 2,0}(x)\right) \\
= & \sum_{l=0}^{-M-k} \frac{(-M-k) !}{l !(-M-k-l) !} D^{-M-k-l}\left(x^{-k}\right) D^{l}\left(e^{-x / 2} x^{-1 / 2} W_{M / 2+N / 2+1 / 2,0}(x)\right) \\
= & \sum_{l=0}^{-M-k} \frac{(-M-k) !(-1)^{-M-k}(-M-l-1) !}{l !(-M-k-l) !(k-1) !} \\
& \cdot x^{M-1 / 2+l / 2} e^{-x / 2} W_{M / 2+N / 2+1 / 2+l / 2 ; l / 2}(x)
\end{aligned}
$$


Substituting (12) into (11) and interchanging the order of summations, we obtain in place of (11) the simpler form

$$
\sum_{l=0}^{-M-k} \frac{(-M) !}{l !(-M-l)} C_{l}(-M) x^{M / 2+l / 2} W_{M / 2+N / 2+1 / 2+l / 2, l / 2}(x),
$$

where $M \leqq N \leqq-M$ and $C_{l}(-M)$ can be written in terms of the Gauss function

$$
C_{l}(-M)=1-{ }_{2} F_{1}(-M-l,-M / 2-N / 2 ;-M ; 1) .
$$

Consequently, we have

$$
\begin{aligned}
\frac{\partial}{\partial \kappa} W_{\kappa, M / 2}(x) & \left.\right|_{k=N / 2+1 / 2} \\
= & \sum_{l=0}^{-M-1} \frac{(-M) ! C_{l}(-M)}{l !(M-l)} x^{M / 2+l / 2} W_{M / 2+N / 2+1 / 2+l / 2, l / 2}(x) \\
& +\sum_{k=1}^{N / 2-M / 2} \frac{(-1)^{k}(N / 2-M / 2) !}{k(N / 2-M / 2-k) !} W_{N / 2+1 / 2-k, M / 2}(x)+W_{N / 2+1 / 2, M / 2}(x) \log x
\end{aligned}
$$

for $M \leqq N \leqq-M, M$ and $N$ integers of like parity, and with $C_{l}(-M)$ given by (14). Analogously,

$$
\begin{aligned}
\frac{\partial}{\partial \kappa} & \left.W_{\kappa, M / 2}(x)\right|_{\kappa=N / 2+1 / 2} \\
= & \sum_{k=1}^{N / 2+M / 2} \frac{(-1)^{k}(N / 2+M / 2) !}{k(N / 2+M / 2-k) !} W_{N / 2+1 / 2-k, M / 2}(x) \\
& +\sum_{l=0}^{M-1} \frac{M ! C_{l}(M)}{l !(M-l)} x^{-M / 2+l / 2} W_{-M / 2+N / 2+1 / 2+l / 2, l / 2}(x)+W_{N / 2+1 / 2, M / 2}(x) \log x
\end{aligned}
$$

for $-M \leqq N \leqq M, M$ and $N$ integers of like parity, and with $C_{l}(M)$ given by (14).

For $N \leqq M \leqq-N$, both sums of (8) must be replaced by sums which involve the $G_{23}^{30}$-function; reduction to the Whittaker function does not seem to be possible, except when one of the equalities holds, cases covered already by (15) and (16).

3. Partial Differentiation of $W_{x, \mu}(x)$ with Respect to the Second Parameter and of $K_{v}(x)$ with Respect to Order. An appropriate analog of (5) for differentiation with respect to $\mu$, again setting $\kappa=N / 2+\frac{1}{2}$ and $\mu=M / 2$, seems to be

$$
\begin{aligned}
& \mathscr{M}\left\{\left.\left(\partial / \partial_{\mu}\right) W_{N / 2+1 / 2, \mu}(x)\right|_{\mu}=M / 2\right. \\
&=g(s)\left(\psi \left(M / 2+\frac{1}{2}\right.\right.+s)-\psi\left(-N / 2+\frac{1}{2}+s\right) \\
&\left.+\psi\left(-N / 2+\frac{1}{2}+s\right)-\psi\left(-M / 2+\frac{1}{2}+s\right)\right),
\end{aligned}
$$

in which merely addition and subtraction of suitable terms is used to produce 
differences involving the $\psi$-function so that (1) can be applied. An analog of (8) is easily obtained in the form

$$
\begin{aligned}
\left.\frac{\partial}{\partial \mu} W_{N / 2+1 / 2, \mu}(x)\right|_{\mu=M / 2}= & \sum_{k=1}^{N / 2+M / 2} \frac{(-1)^{k-1}(N / 2+M / 2) !}{k(N / 2+M / 2-k) !} W_{N / 2+1 / 2-k, M / 2}(x) \\
& +\sum_{k=1}^{N / 2-M / 2} \frac{(-1)^{k}(N / 2-M / 2) !}{k(N / 2+M / 2-k) !} W_{N / 2+1 / 2-k, M / 2}(x)
\end{aligned}
$$

for $-N \leqq M \leqq N, M$ and $N$ integers of like parity. Analogs of (15) and (16) can also be written down directly, but, again, the case $N \leqq M \leqq-N$ seems not to be representable in terms of Whittaker functions. This is somewhat disappointing in view of the possibility of connecting these formulas to the partial derivative with respect to order of the Bessel function of the third kind by means of the relation

$$
K,(x / 2)=(\pi / x)^{1 / 2} W_{0, v}(x) .
$$

The formula

$$
\left.\frac{\partial}{\partial \nu} K_{\nu}(x)\right|_{,=N}=\frac{N !}{2} \sum_{k=0}^{N-1} \frac{(x / 2)^{k-N}}{k !(N-k)} K_{k}(x)
$$

is given, for example, as formula (9.6.45) on p. 377 of [1]. This can be obtained by similar manipulations of Mellin transforms starting from formula (26), rather than (28), on p. 331 of the tables [3]. Using formula (5) on p. 307 of [3] and using ideas developed in our formulas (9)-(12), we have

$$
\begin{aligned}
& \mathscr{M}\left\{\left.(\partial / \partial \nu) K_{\nu}\left(2 x^{1 / 2}\right)\right|_{\nu=N}\right\} \\
&=2^{-2} \Gamma(N / 2+s) \Gamma(-N / 2+s)(\psi(N / 2+s)-\psi(-N / 2+s)) \\
&=\frac{N !}{2} \sum_{k=1}^{N} \frac{(-1)^{k-1}}{k(N-k) !} \frac{\Gamma(N / 2+s)}{\Gamma(-N / 2+k+s)} \frac{\Gamma^{2}(-N / 2+s)}{2} \\
&= \mathscr{M}\left\{-\frac{N !}{2} \sum_{k=1}^{N} \frac{(-1)^{N}}{k(N-k) !} x^{N / 2} D^{N-k}\left(x^{-k} \cdot K_{0}\left(2 x^{1 / 2}\right)\right)\right\} .
\end{aligned}
$$

If, in a manner analogous to (12), the derivative of order $N-k$ is represented as a sum, the order of summation interchanged, and the inner sum then simplified to the value 1 , we obtain a formula equivalent to (20).

4. Generalizations to the $G$-Function. Since the Mellin transform of the $G$-function is a quotient of products of $\Gamma$-functions, it seems a natural direction for generalization of these partial derivative formulas. The methods used to discover formulas (8), (18), and (20) can be used for the following generalizations.

If $m \geqq 2, \quad n \geqq 0, \quad N_{j} \geqq 0, \quad M_{j} \geqq 0, \quad \alpha>\max _{i \leqq j \leqq n} \operatorname{Re}\left(a_{j}\right)-1$, 


$$
\begin{aligned}
& \begin{aligned}
(1-m) & \left.\frac{\partial}{\partial a_{n+1}} G_{n+1, m+n}^{m, n}\left(x \mid \begin{array}{l}
a_{1}, \cdots, a_{n}, a_{n+1} \\
\alpha+M_{1}, \cdots, \alpha+M_{m}, a_{1}+N_{1}, \cdots, a_{n}+N_{n}
\end{array}\right)\right|_{a_{n+1}=\alpha} \\
= & G_{n+1, m+n}^{m, n}\left(x \mid \begin{array}{l}
a_{1}, \cdots, a_{n}, \alpha \\
\alpha+M_{1}, \cdots, \alpha+M_{m}, a_{1}+N_{1}, \cdots, a_{n}+N_{n}
\end{array}\right) \log x
\end{aligned} \\
& +\sum_{j=1}^{m} \sum_{k_{j}=1}^{M_{j}} \frac{(-1)^{k}\left(M_{j}\right) !}{k_{j}\left(M_{j}-k_{j}\right) !} G_{n+1, m+n}^{m, n} \\
& \quad \cdot\left(\begin{array}{l}
a_{1}, \cdots, a_{n}, \alpha+k \\
\alpha+M_{1}, \cdots, \alpha+M_{m}, a_{1}+N_{1}, \cdots, a_{n}+N_{n}
\end{array}\right) \\
& -\sum_{j=1}^{n} \sum_{k_{j}=1}^{N_{j}} \frac{(-1)^{k_{j}}\left(N_{j}\right) !}{k_{j}\left(N_{j}-k_{j}\right) !} G_{n+1, m+n}^{m, n} \\
& \cdot\left(x \mid \begin{array}{l}
a_{1}, \cdots, a_{n}, \alpha \\
\alpha+M_{1}, \cdots, \alpha+M_{m}, a_{1}+N_{1}, \cdots, a_{j}+N_{j}-k_{j}, \cdots, a_{n}+N
\end{array}\right) .
\end{aligned}
$$

The cases in which the integer parameters $N_{j}$ and $M_{j}$ are not all positive lead to modifications along the lines of the earlier development of formulas (9) through (16). Companion formulas for

$$
\left.\frac{\partial}{\partial b_{m+1}} G_{m+n, m+1}^{m, n}(x)\right|_{b_{m+1}}=\beta
$$

follow by use of formula (9) on p. 209 of [2].

If $M$ and $N$ are of the same parity, $-N \leqq M \leqq N, m \geqq 2, n<p$, then

$$
\begin{aligned}
\frac{\partial}{\partial \nu} G_{p, q}^{m, n}(x & \left.\mid \begin{array}{l}
a_{1}, \cdots, a_{p-1},-N / 2 \\
\nu,-\nu, b_{3}, \cdots, b_{q}
\end{array}\right)\left.\right|_{\nu=M / 2} \\
(24)= & \sum_{k=1}^{N / 2+M / 2} \frac{(-1)^{k-1}(N / 2+M / 2) !}{k(N / 2+M / 2-k) !} G_{p, q}^{m, n}\left(x \mid \begin{array}{l}
a_{1}, \cdots, a_{p-1},-N / 2+k \\
M / 2,-M / 2, b_{3}, \cdots, b_{q}
\end{array}\right) \\
& +\sum_{k=1}^{N / 2-M / 2} \frac{(-1)^{k-1}(N / 2-M / 2) !}{k(N / 2-M / 2-k) !} G_{p, q}^{m, n}\left(x \mid \begin{array}{l}
a_{1}, \cdots, a_{p-1},-N / 2+k \\
M / 2,-M / 2, b_{3}, \cdots, b_{q}
\end{array}\right) .
\end{aligned}
$$

If $m \geqq 2, N \geqq 0$, we obtain

$$
\begin{aligned}
\frac{\partial}{\partial \nu} G_{p, q}^{m, n} & \left.\left(x \mid \begin{array}{l}
a_{1}, \cdots, a_{p} \\
\nu / 2,-\nu / 2, b_{3}, \cdots, b_{q}
\end{array}\right)\right|_{v=N} \\
(25) & =\frac{N !}{2} \sum_{k=1}^{N} \frac{(-1)^{N-1} x^{N / 2}}{k(N-k) !} D^{N-k}\left(x^{N / 2-k} G_{p, q}^{m, n}\left(x \mid \begin{array}{l}
a_{1}, \cdots, a_{p} \\
-N / 2,-N / 2, b_{3}, \cdots, b_{q}
\end{array}\right)\right) \\
& =\frac{N !}{2} \sum_{l=0}^{N-1} \frac{1}{l !(N-l)} G_{p, q}^{m, n}\left(x \mid \begin{array}{l}
a_{1}, \cdots, a_{p} \\
l-N / 2,-N / 2, b_{3}, \cdots, b_{q}
\end{array}\right) .
\end{aligned}
$$

In these formulas, it should be noted that forms somewhat different in appearance can be obtained by the use of the relations

$$
x^{\sigma} G_{p, q}^{m, n}\left(x \mid \begin{array}{l}
a_{1}, \cdots, a_{p} \\
b_{1}, \cdots, b_{q}
\end{array}\right)=G_{p, q}^{m, n}\left(x \mid \begin{array}{l}
a_{1}+\sigma, \cdots, a_{p}+\sigma \\
b_{1}+\sigma, \cdots, b_{q}+\sigma
\end{array}\right),
$$




$$
G_{p, q}^{m, n}\left(x^{-1} \mid \begin{array}{l}
a_{1}, \cdots, a_{p} \\
b_{1}, \cdots, b_{q}
\end{array}\right)=G_{q, p}^{n, m}\left(x \mid \begin{array}{l}
1-b_{1}, \cdots, 1-b_{q} \\
1-a_{1}, \cdots, 1-a_{p}
\end{array}\right) .
$$

Among the special cases of (22), (24), and (25) other than (8), (18), and (20), we note that if we set $M=N=1, m=q=3, n=0, p=2, a_{1}=\frac{1}{2}$, $b_{3}=-\frac{1}{2}$ in $(24)$, we have

$$
\left.\frac{\partial}{\partial \nu} K_{\nu}(x / 2)\right|_{\nu=1 / 2}=-(\pi / x)^{1 / 2} e^{x / 2} \operatorname{Ei}(-x),
$$

which is equivalent to formula (10.2.34) on p. 445 of [1].

As a further example, we display a formula involving the hypergeometric function of Gauss, from which other special cases can be obtained. By use of (26) with (25) if we let $m=p=q=2, n=1, a_{1}=-\sigma, a_{2}=c-\sigma, N \geqq 0$, $\sigma-N / 2$ not zero or a negative integer, we have

$$
\begin{aligned}
\frac{\partial}{\partial \nu}{ }_{2} F_{1}(\sigma & +\nu / 2, \sigma-\nu / 2 ; c ; z)\left.\right|_{\nu=N} \\
= & \frac{N !}{2} \sum_{l=0}^{N-1} \frac{\Gamma(l+\sigma-N / 2)}{l !(N-l) \Gamma(\sigma+N / 2)}{ }_{2} F_{1}(l+\sigma-N / 2, \sigma-N / 2 ; c ; z) \\
& -\frac{1}{2}(\psi(\sigma+N / 2)-\psi(\sigma-N / 2))_{2} F_{1}(\sigma+N / 2, \sigma-N / 2 ; c ; z) .
\end{aligned}
$$

Since the hypergeometric function is defined by a series, we could alternatively derive (29) by manipulations on the series which are analogs of those used on the Mellin transforms. To illustrate this, we consider

$$
\begin{aligned}
S= & \left.\frac{\partial}{\partial \nu}\left(\frac{\Gamma(\sigma+\nu / 2) \Gamma(\sigma-\nu / 2)}{\Gamma(c)}{ }_{2} F_{1}(\sigma+\nu / 2, \sigma-\nu / 2 ; c ; u)\right)\right|_{\nu=N} \\
= & \sum_{n=0}^{\infty} \frac{\Gamma(\sigma+N / 2+n) \Gamma(\sigma-N / 2+n)}{\Gamma(c+n)} \\
& \cdot \frac{u^{n}}{n !} \frac{1}{2}(\psi(\sigma+N / 2+n)-\psi(\sigma-N / 2+n))
\end{aligned}
$$

so that, if we apply formula (1) and interchange the order of the summations, we obtain

$$
\begin{aligned}
S= & \frac{N !}{2} \sum_{k=1}^{N} \frac{(-1)^{k-1}}{k(N-k) !} \sum_{n=0}^{\infty} \frac{\Gamma(\sigma+N / 2+n) \Gamma^{2}(\sigma-N / 2+n)}{\Gamma(c+n) \Gamma(\sigma-N / 2+k+n)} \frac{u^{n}}{n !} \\
= & \frac{N !}{2} \sum_{k=1}^{N} \frac{(-1)^{k-1} u^{-\sigma-N / 2}}{k(N-k) !} \\
& \cdot \sum_{n=0}^{\infty} \frac{\Gamma^{2}(\sigma-N / 2+n)}{\Gamma(c+n)}(\sigma-N / 2+n+k)_{N-k} \frac{u^{n+\sigma+N / 2}}{n !} \\
=\frac{N !}{2} \sum_{k=1}^{N} \frac{(-1)^{k-1} u^{-\sigma-N / 2}}{k(N-k) !} & \cdot\left(u^{2} D_{u}\right)^{N-k}\left(u^{k} \cdot u^{\sigma-N / 2} F_{1}(\sigma-N / 2, \sigma-N / 2 ; c ; u)\right) .
\end{aligned}
$$


If we next expand the differential operator $\left(u^{2} D_{u}\right)^{N-k}$ which is applied to the product, we have

$$
\begin{aligned}
& \left(u^{2} D_{u}\right)^{N-k}\left(u^{k} \cdot u^{\sigma-N / 2} F_{1}(\sigma-N / 2, \sigma-N / 2 ; c ; u)\right) \\
& \quad=\sum_{l=0}^{N-k}\left(\begin{array}{c}
N-k \\
l
\end{array}\right)\left(u^{2} D_{u}\right)^{N-k-l}\left(u^{k}\right) \cdot\left(u^{2} D_{u}\right)^{l}\left(u^{\sigma-N / 2}{ }_{2} F_{1}(\sigma-N / 2, \sigma-N / 2 ; c ; u)\right) \\
& (32) \quad \\
& =\sum_{l=0}^{N-k}\left(\begin{array}{c}
N-k \\
l
\end{array}\right) \frac{(N-l-1) !}{(k-1) !} u^{N-l} \cdot u^{\sigma-N / 2+l}{ }_{2} F_{1}(l+\sigma-N / 2, \sigma-N / 2 ; c ; u) .
\end{aligned}
$$

The substitution from (32) into (31), a change of the order of the summations, and simplification gives us

$$
S=\sum_{l=0}^{N-1} \frac{\Gamma(\sigma-N / 2) \Gamma(l+\sigma-N / 2)}{l !(N-l) \Gamma(c)}{ }_{2} F_{1}(l+\sigma-N / 2, \sigma-N / 2 ; c ; u) .
$$

With the expansion of the partial derivative in (30), we see that (29) can then be obtained.

As a final example, we note that ${ }_{2} F_{1}$ can be expressed in terms of Gegenbauer functions $C_{a}^{v}$ (see Section 3.15) of [2]), so that (29) can be rewritten

$$
\begin{aligned}
\left.\frac{\partial}{\partial \nu} C_{-\sigma+\nu / 2}^{\sigma}(y)\right|_{\nu=N}= & -\frac{1}{2}(\psi(1-\sigma+N / 2)-\psi(\sigma-N / 2)) C_{-\sigma+N / 2}^{\sigma}(y) \\
& +\frac{N !}{2} \sum_{l=0}^{N-1} \frac{\Gamma(l+2 \sigma-N)}{l !(N-l) \Gamma(2 \sigma)} C_{-\sigma+N / 2}^{\sigma-N / 2+l / 2}(y),
\end{aligned}
$$

for $\sigma-N / 2$ not an integer and $N \geqq 0$; also in terms of Legendre functions $\left.\frac{\partial}{\partial \nu} P_{\nu / 2-1 / 2}^{1 / 2-\sigma}(y)\right|_{\nu=N}=-\frac{1}{2}(\psi(\sigma+N / 2)-\psi(\sigma-N / 2)) P_{N / 2-1 / 2}^{1 / 2-\sigma}(y)$

$$
\begin{array}{r}
+\frac{N !}{2} \sum_{l=0}^{N-1} \frac{2^{l / 2-N / 2} \Gamma(l+\sigma-N / 2) \Gamma(l / 2-N / 2+\sigma+1 / 2)}{l !(N-l) \Gamma(\sigma+N / 2) \Gamma(\sigma+1 / 2)} \\
\cdot\left(y^{2}-1\right)^{N / 4-l / 4} P_{l / 2-1 / 2}^{1 / 2-\sigma+N / 2-l / 2}(y)
\end{array}
$$

for $\sigma-N / 2$ not zero or a negative integer and $N \geqq 0$.

Department of Mathematics

University of Wyoming

Laramie, Wyoming 82071

1. M. Abramowitz \& I. A. Stegun (Editors), Handbook of Mathematical Functions, With Formulas, Graphs, and Mathematical Tables, Nat. Bur. Standards Appl. Math. Series, 55, Superintendent of Documents, U.S. Government Printing Office, Washington, D.C., 1964. MR 29 \# 4914.

2. A. Erdélyi, W. Magnus, F. Oberhettinger \& F. G. Tricomi, Higher Transcendental Functions. Vols. 1, 2, McGraw-Hill, New York, 1953. MR 15, 419.

3. A. Erdélyi, W. Magnus, F. Oberhettinger \& F. G. Tricomi, Tables of Integral Transforms. Vol. 1, McGraw-Hill, New York, 1954. MR 15, 868.

4. Bernard J. LaURenzi, "Derivatives of Whittaker functions $W_{k, 1 / 2}$ and $M_{k, 1 / 2}$ with respect to order $k$," Math. Comp., v. 27, 1973, pp. 129-132.

5. L. J. Slater, Confluent Hypergeometric Functions, Cambridge Univ. Press, New York, 1960. MR 21 \# 5753. 